\title{
RVFL-Based Optical Fiber Intrusion Signal Recognition With Multi-Level Wavelet Decomposition as Feature
}

\author{
Yanping $\mathrm{WANG}^{1}$, Dianjun $\mathrm{GONG}^{1}$, Liping $\mathrm{PANG}^{2 *}$, and Dan $\mathrm{YANG}^{1}$ \\ ${ }^{1}$ School of Electronic and Information Engineering, North China University of Technology, Beijing 100144, China \\ ${ }^{2}$ School of Aviation Science and Engineering, Beijing University of Aeronautics and Astronautics, Beijing 100191, China \\ *Corresponding author: Liping PANGＥ-mail: pangliping@buaa.edu.cn
}

\begin{abstract}
The optical fiber pre-warning system (OFPS) has been gradually considered as one of the important means for pipeline safety monitoring. Intrusion signal types are correctly identified which could reduce the cost of troubleshooting and maintenance of the pipeline. Most of the previous feature extraction methods in OFPS are usually quested from the view of time domain. However, in some cases, there is no distinguishing feature in the time domain. In the paper, firstly, the intrusion signal features of the running, digging, and pick mattock are extracted in the frequency domain by multi-level wavelet decomposition, that is, the intrusion signals are decomposed into five bands. Secondly, the average energy ratio of different frequency bands is obtained, which is considered as the feature of each intrusion type. Finally, the feature samples are sent into the random vector functional-link (RVFL) network for training to complete the classification and identification of the signals. Experimental results show that the algorithm can correctly distinguish the different intrusion signals and achieve higher recognition rate.
\end{abstract}

Keywords: OFPS; multi-level wavelet decomposition; optical fiber signal recognition; RVFL

Citation: Yanping WANG, Dianjun GONG, Liping PANG, and Dan YANG, "RVFL-Based Optical Fiber Intrusion Signal Recognition With Multi-Level Wavelet Decomposition as Feature," Photonic Sensors, 2018, 8(3): 234-241.

\section{Introduction}

At present, the pipeline transportation is one of the main modes of transportation for oil and natural gas. However, oil or natural gas leakage caused by pipeline damage can lead to serious environmental pollution and economic loss [1-3]. In response to this problem, some researches on pipeline detection have been proposed in domestic and international [4]. The optical fiber pre-warning system (OFPS) is often used for disasters occurrence monitoring such as oil and gas pipeline leakage, and it is mainly used for detection and recognition of intrusion source
$[5,6]$. Some documents proposed they found an effective way to set the soft or hard thresholds for every point along the fiber adaptively to improve the signal-to-noise ratio (SNR) [7, 8]. With different background noises, the constant false alarm rate (CFAR) algorithm is exploited to achieve an optimal adaptive threshold detection $[9,10]$.

Above-mentioned methods use the adaptive threshold to improve the detection efficiency and the system pre-warning performance, but the false alarm problem is still more prominent. For example, the vibration of passing vehicle makes no direct damage to the pipeline, but this kind of vibration can still

Received: 1 March 2018 / Revised: 24 May 2018

(C) The Author(s) 2018. This article is published with open access at Springerlink.com

DOI: $10.1007 / \mathrm{s} 13320-018-0496-7$

Article type: Regular 
cause false alarm, which will lead to wasting of system resources. One of the key technologies of the OFPS is to accurately distinguish the harmful and harmless intrusion event. Moreover, the identification of harmful intrusion signals is able to indicate for us the intrusion points. Therefore, we mainly focus on the identification of the intrusion types based on the distinction between harmful and harmless signals in OFPS in this paper.

Regarding to the feature extraction of the signal in OFPS, a feature extraction method for optical fiber signals in the time domain is quite effective in distinguishing among harmful and harmless intrusion signals [11]. However, for identifying different types of harmful intrusion events, it performs badly. In our previous experiment, the intrusion signals in the time domain were transformed into the frequency domain by wavelet transform, and it was found that the frequency spectrum distribution of each harmful intrusion signal was quite different. Hence, we analyze the problems in the frequency domain in this paper, and the ultimate extracted feature in the frequency domain is input into random vector functional-link (RVFL) classifier. The input weights and biases of RVFL are chosen randomly according to the uniform distribution in a given range. The network uses linear regression method instead of multiple iterative optimizations. The above measures greatly reduce the time consumption for training the networks. The wavelet packet decomposition is a method to recognize the intrusion signals, and its experimental results show that intrusion signals, such as gas leakage, artificial excavation, and walking, can be effectively distinguished [12]. However, during the processing, plenty of wavelet coefficients are generated by the wavelet packet decomposition, which can induce the complexity of training network, since it is directly proportional to the number of training samples. It is known that the multi-level wavelet decomposition has the nature with fewer wavelet coefficients than the wavelet packet decomposition [13, 14]. Therefore, it is more suitable for the neural networks classification. However, the wavelet coefficients obtained by the multi-level wavelet decomposition cannot be directly input into the network as features. So we need to adopt an effective feature extraction method. The battlefield-acoustic signal is decomposed, and the energy ratio is taken as the characteristic vector for classification, where the performance of classifying the type of battlefield-acoustic signal is obvious [15]. An improved fast Fourier transform (FFT) filtering method based on the energy-ratio pretreatment can distinguish the harmful intrusion event and harmless interference event efficiently [16]. In this paper, the energy ratio feature extraction method is utilized, which can preserve the frequency band information with low cost of calculation. This energy ratio of different frequency bands by multi-layer wavelet decomposition is used as the feature sample, and the effective information is retained. At last, the extracted feature data are input into the RVFL neural network for training and classification.

The real field experiment is conducted to verify the proposed algorithm. The experimental results show that this algorithm can effectively recognize the running, digging, and pick mattock signals.

\section{Algorithm flowchart of feature extraction and classification}

Figure 1 shows the processing flow of this algorithm. First of all, we detect three intrusion signals and collect data. Furthermore, these signals are decomposed by discrete wavelet transform to obtain the wavelet coefficients in six frequency bands, where the average energy ratio of wavelet coefficients in each scale is calculated, respectively. Then, we remain the average energy ratio of the five bands as an independent feature. Finally, the intrusion signal samples of features are inputted into an RVFL network for training and testing. 


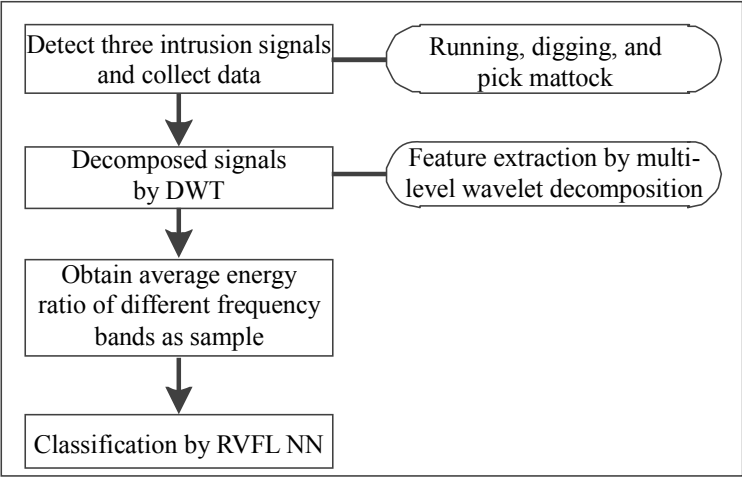

Fig. 1 Processing flow of intrusion signal recognition.

\subsection{Feature extraction from multi-level wavelet decomposition}

The fundamental strategy for the wavelet transform as a time-frequency transform method is decomposition-reconstruction. Namely, the original signal can be decomposed into a wavelet series that can be further used to reconstruct the original one. Moreover, the wavelet series is actually somewhat features of the original signal but more obvious than it. The basis function of wavelet transform has both scales and displacements transformations.

The discrete wavelet transform (DWT) of signal $s(t)$ is defined as

$$
\xi(\alpha, \tau)=\frac{1}{\sqrt{2^{\alpha}}} \int_{-\infty}^{+\infty} s(t) \psi\left(\frac{t}{2^{\alpha}}-\tau\right) d t
$$

where $\psi(t)$ is the mother wavelet function, $\alpha$ is the scale factor, and $\tau$ provides the displacement information of mother function. Hence, the multi-level wavelet decomposition is achieved along with different scales and displacements. The transformation process is described in Fig. 2.

As shown in Fig. 2, LA represents low frequency, and HD represents high frequency. This decomposition is the process of retaining high frequency and subdividing the low frequency. In this paper, the signal $s(t)$ is decomposed into five layers by DWT, and the low frequency part $\mathrm{LA}_{5}$ and the high frequency parts $\mathrm{HD}_{5}-\mathrm{HD}_{1}$ are obtained. Then the average energy in each frequency band is obtained as

$$
E_{i}=\sum_{n=1}^{N}\left[\xi_{i}(n)\right]^{2} / N, i=1,2,3, \cdots, 6
$$

where $\xi_{i}(n)$ is the wavelet coefficient in the $i$ th frequency band, and $N$ is the length of the wavelet coefficient in each frequency band. Then, the total energy can be accumulated by all frequency bands as expressed as follows:

$$
E_{\text {sum }}=\sum_{i=1}^{6} E_{i} .
$$

Therefore, the average energy ratio $\Omega_{i}$ in each frequency band is given as follows:

$$
\Omega_{i}=E_{i} / E_{\text {sum }}, i=1,2,3, \cdots, 6 .
$$

We find that the $\mathrm{HD}_{1}$ frequency band of three intrusion signals have not obvious difference with others. Hence, we artificially remove the $\mathrm{HD}_{1}$ and retain average energy ratios of remaining five frequency bands. Then, we take it into RVFL as independent feature of samples, and the final feature sample is $\Omega=\left\{\Omega_{1}, \Omega_{2}, \Omega_{3}, \Omega_{4}, \Omega_{5}\right\}$.

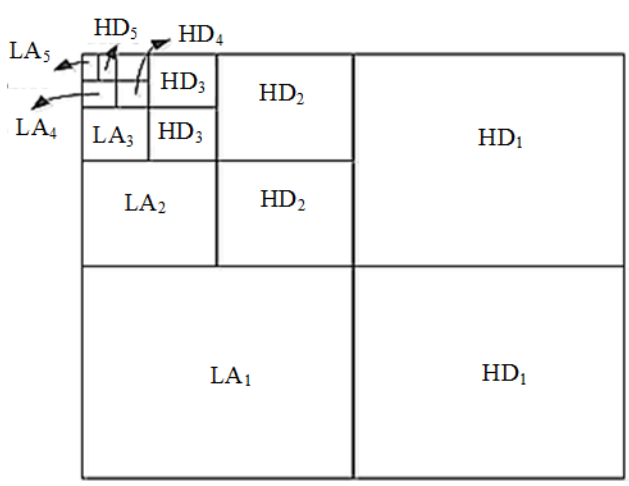

Fig. 2 Structure of five-layer wavelet decomposition.

\subsection{RVFL NN classification}

The RVFL neural network can overcome the difficulty of slow convergence and local minimum inherently in the traditional gradient-based learning algorithms. Besides, the RVFL has direct connections between the input layer and the output layer, thus its weights no longer need updating during learning as shown in Fig. 3. We calculate the network weights of nodes connected with the output layer by solving simple linear regression problems with the random input weights $w$ and biases $b$.

According to the neural network calculation formula and the unique structure of RVFL, the function $\hat{y}$ is given as 


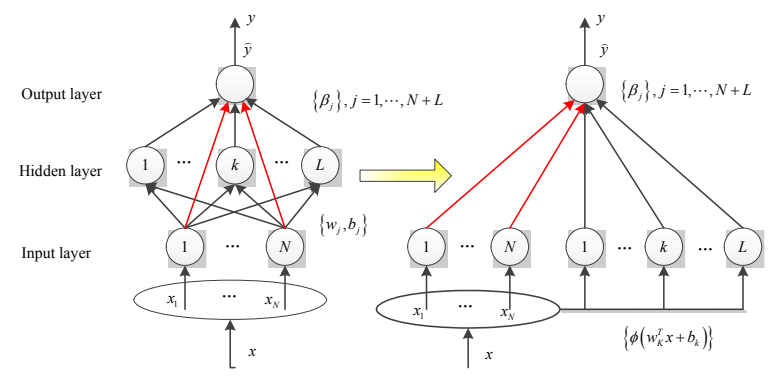

Fig. 3 Structure of the RVFL NN.

$$
\widehat{y}=\sum_{k=1}^{L} \beta_{k} \phi\left(w_{k}^{T} x+b_{k}\right)
$$

where $w_{k}$ is the weight value from the input layer to hidden layer, $\beta_{k}$ is the weight value from the hidden layer to output layer, $w_{k}^{T} x+b_{k}$ is the linear transformation of the input vector, and $\phi(\cdot)$ is the basis function. In this network, the weight $w_{k}$ and biases $b_{k}$ are randomly set in the learning process. Hence, the weight $\beta_{k}$ is the only parameter during learning resulting in a high efficiency.

In this paper, we use the mean square error as the objective function. So the learning problem can be formulated as

$$
E_{\beta}=\min _{\beta} \sum_{i=1}^{N}\left[f\left(x_{i}\right)-\sum_{k=1}^{L} \beta_{k} \phi\left(x_{i} ; w_{k}, b_{k}\right)\right]^{2}
$$

which can be rewritten as

$$
E_{\beta}=\min _{\boldsymbol{\beta}}\|\mathbf{Y}-\boldsymbol{\Phi} \boldsymbol{\beta}\|_{2}^{2}
$$

where $\boldsymbol{\beta}$ is the matrix form of $\beta_{k}$ and $\boldsymbol{\Phi}$ can be written as

$$
\boldsymbol{\Phi}=\left[\begin{array}{ccc}
\phi\left(x_{1} ; w_{1}, b_{1}\right) & \cdots & \phi\left(x_{1} ; w_{L}, b_{L}\right) \\
\vdots & \ddots & \vdots \\
\phi\left(x_{N} ; w_{1}, b_{1}\right) & \cdots & \phi\left(x_{N} ; w_{L}, b_{L}\right)
\end{array}\right], \mathbf{Y}=\left[\begin{array}{c}
y_{1} \\
y_{2} \\
\vdots \\
y_{N}
\end{array}\right]
$$

According to the definition of matrix 2-Norm, (7) becomes as follows:

$$
E_{\beta}=\min _{\boldsymbol{\beta}}(\mathbf{Y}-\boldsymbol{\Phi} \boldsymbol{\beta})^{T}(\mathbf{Y}-\boldsymbol{\Phi} \boldsymbol{\beta}) .
$$

Taking the derivative of (8), then we get

$$
\frac{\partial E_{\beta}}{\partial \boldsymbol{\beta}}=2 \boldsymbol{\Phi}^{T}(\boldsymbol{\Phi} \boldsymbol{\beta}-\mathbf{Y})
$$

where $\boldsymbol{\Phi}^{T} \boldsymbol{\Phi}$ is a full rank or positive-definite matrix. Let (9) be zero, then $\boldsymbol{\beta}$ can be obtained as follows:

$$
\boldsymbol{\beta}=\left(\boldsymbol{\Phi}^{T} \boldsymbol{\Phi}\right)^{-1} \boldsymbol{\Phi}^{T} \mathbf{Y} .
$$

The RVFL network structure can be determined by obtaining the weight $\boldsymbol{\beta}$ from training. Then, the test samples are input into the RVFL network to complete classification.

\section{Experiments on feature extraction and intrusion signal recognition}

\subsection{Collection of measured data}

The measured data were collected in Mentougou District, Beijing, China. The obtained signals are running, digging, and pick mattock by using the CFAR detection. The resolutions in the time and spatial dimensions are $1 \mathrm{~ms}$ and $1 \mathrm{~m}$.

Figure 4 shows that the abscissa represents the position, and the ordinate represents the time. We can see that continuity points are intrusion signals when the intrusion location is about $20 \mathrm{~m}$ and $80 \mathrm{~m}$.
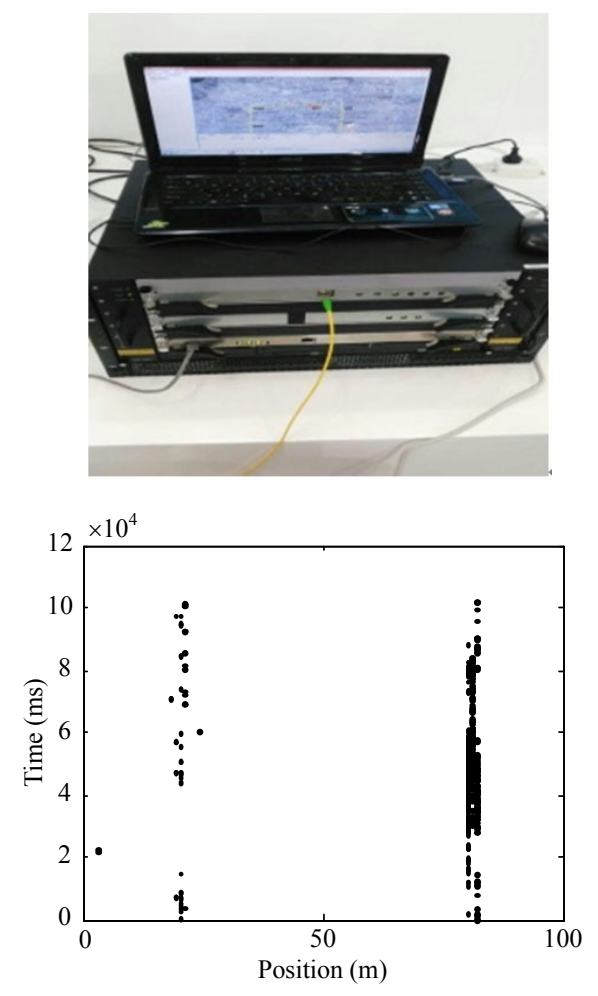

Fig. 4 Detection result of optical vibration signal.

\subsection{Feature extraction of intrusion signal}

The original waveforms of running, digging, and pick mattock signals in the time domain are shown in Fig. 5. According to the method of multi-layer 
wavelet decomposition, the signal frequency spectra are shown in Fig. 6.

We realize the 5-level decomposition of running, digging, and pick mattock signals by using dB3 wavelet, and the results are shown in Fig. 6, wherein $s$ is the original function, ca5 and cd1-cd5 are the wavelet coefficients in different frequency bands. We find that the wavelet coefficients of each layer have a significant difference except cd1. Therefore, we neglect the cd1 in order to reduce the feature dimension. And the remaining wavelets coefficients are used as independent features to distinguish the running, digging, and pick mattock signals. Then, we calculate their energy ratios as shown in Fig. 7.

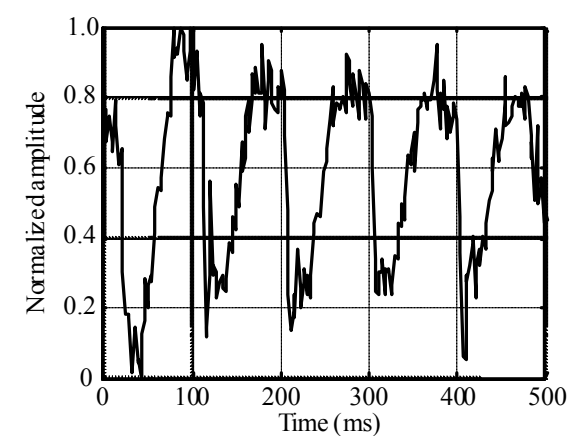

(a)

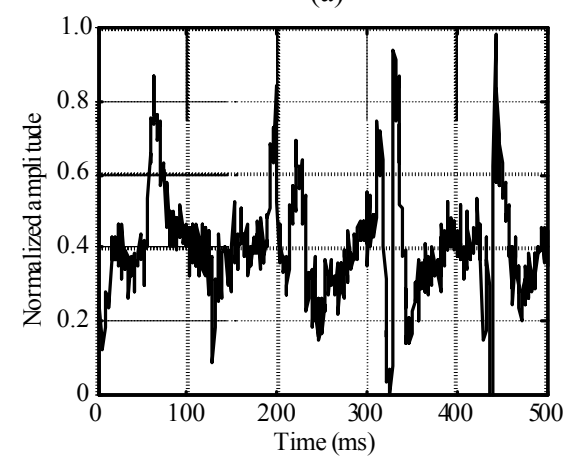

(b)

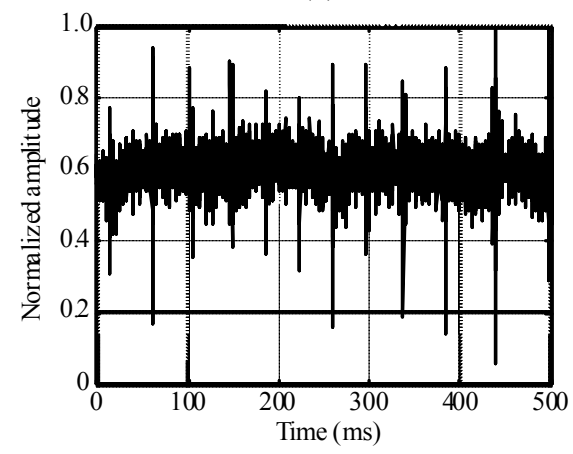

(c)

Fig. 5 Original waveforms of vibration signal: (a) running signals, (b) digging signals, and (c) pick mattock signals.

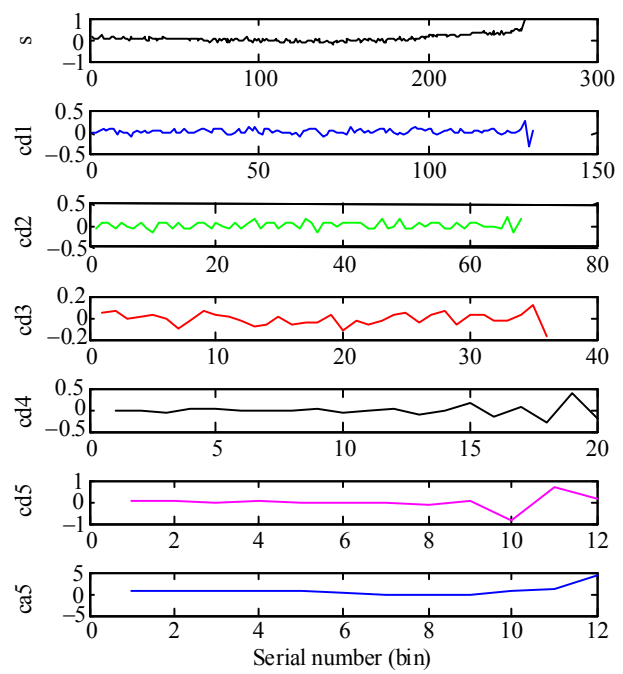

(a)

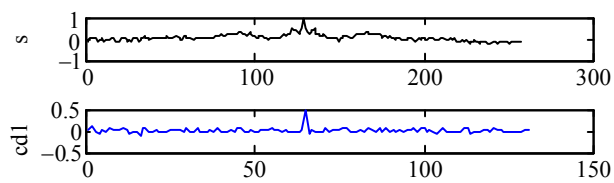

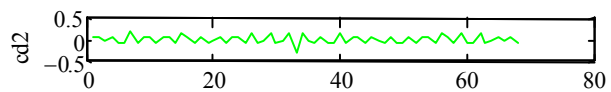

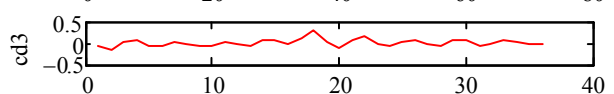

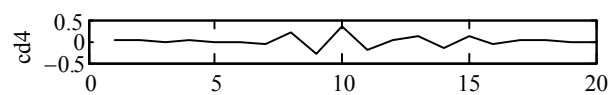

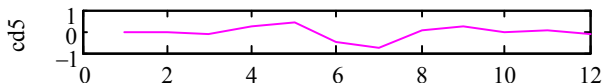

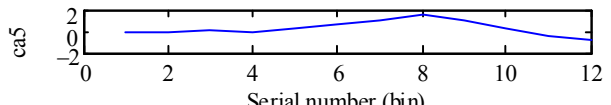

(b)
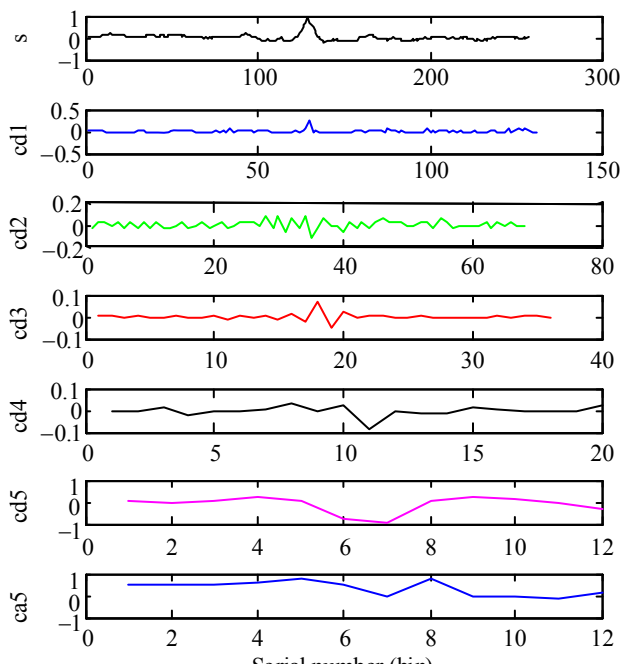

(c)

Fig. 6 Result of multi-level wavelet decomposition: (a) running signals, (b) digging signals, and (c) pick mattock signals. 


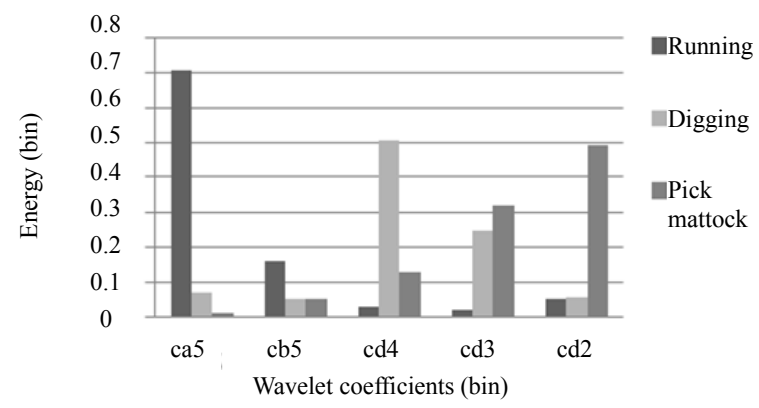

Fig. 7 Energy ratio of wavelet decomposition coefficient for intrusion signals.

It can be seen from Fig. 7 that the energy ratio has significant differences regarding to three kinds of intrusion signals. The energy of running signal is concentrated in the low frequency part ca5, while the energy of digging signal in the intermediate frequency cd4 accounts for the highest one. Moreover, the energy of pick mattock signal is concentrated in the high frequency part cd2. So, we use the energy ratio as samples for the intrusion signal classification by RVFL NN.

\subsection{Identification of intrusion signal by RVFL NN}

In the experiments, we have totally three kinds of intrusion samples including running, digging, and pick mattock, which are trained in pairs labeled with digital numbers 0 and 1, respectively. For example, we set the running signal samples with label 1 while both the digging signal and the pick mattock signal are set with label 0 together. Training samples are used to train to find a proper weight $\boldsymbol{\beta}$. The test samples are input into the RVFL NN model obtained by training and the test results are shown in Fig. 8. It can be clearly seen that the recognition of the running signals is completed after training. We repeat the above processing flow on the other two types of intrusion signals. Finally, all the three types of intrusion signals are classified successfully.

In Fig. 8, the recognition threshold is set to be 0.5 and the samples with recognition results larger than 0.5 will be regarded as the running signals. Otherwise, the samples will be regarded as the digging and pick mattock signals. Similarly, the same recognition strategy is performed on the digging signal as shown in Fig. 8(b). Finally, we have finished the classification for the intrusion signals. To make further accuracy analysis, we calculate the RVFL NN recognition rate of the test sample as shown in Table 1.

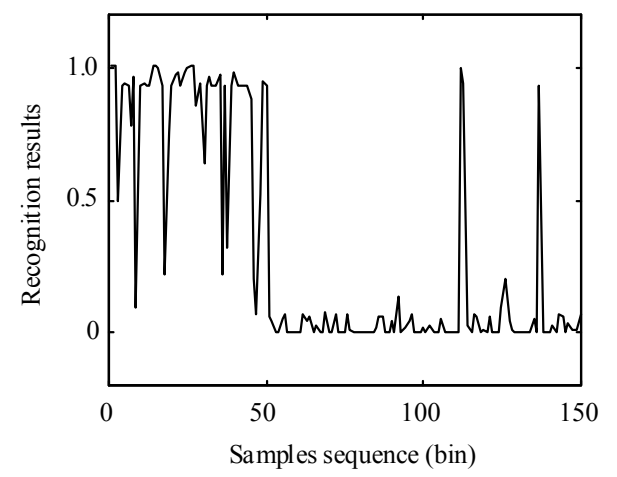

(a)

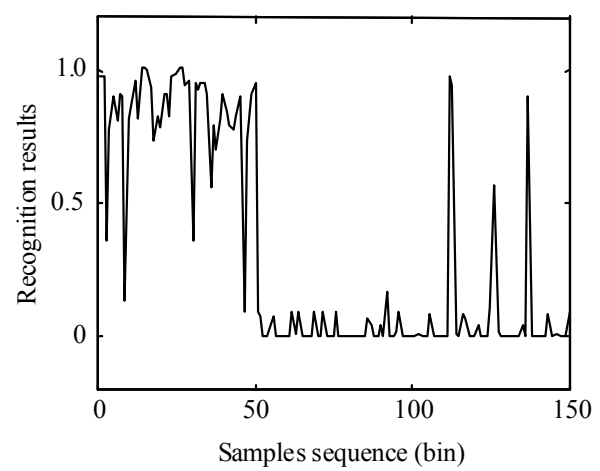

(b)

Fig. 8 Recognition result of RVFL NN: (a) classification results of running signals and (b) classification results of digging signals.

Table 1 Recognition rate of RVFL NN.

\begin{tabular}{|c|c|c|c|c|}
\hline $\begin{array}{l}\text { Real } \\
\text { signals }\end{array}$ & $\begin{array}{l}\text { Running } \\
\text { signal }\end{array}$ & $\begin{array}{l}\text { Digging } \\
\text { signal }\end{array}$ & $\begin{array}{c}\text { Pick mattock } \\
\text { signal }\end{array}$ & $\begin{array}{l}\text { Recognition } \\
\text { accuracy }(\%)\end{array}$ \\
\hline Running signal & 0.92 & 0.08 & 0 & 92 \\
\hline Digging signal & 0.06 & 0.94 & 0 & 94 \\
\hline $\begin{array}{l}\text { Pick mattock } \\
\text { signal }\end{array}$ & 0.02 & 0.02 & 0.96 & 96 \\
\hline
\end{tabular}

It can be seen from the Table 1 that the method provided in this paper can effectively identifies the running signal, the digging signal, and the pick mattock signal in the optical fiber in the OFPS and achieves high recognition rate which is $92 \%, 94 \%$, and $96 \%$, respectively.

\section{Conclusions}

In this paper, the recognition of vibration signal is studied. Firstly, we extract intrusion signals in the 
frequency domain by the five-level wavelet decomposition. Then, according to the characteristics of different vibration signals, the energy ratio feature extraction method is drawn on. After that, we calculate the average energy ratio as sample into the RVFL NN to complete the identification and classification. The recognition rates of the running signal, the digging signal, and the pick mattock signal are $92 \%, 94 \%$, and $96 \%$, respectively. The field experiment results show that we can correctly identify the intrusion signals, which verifies the feasibility and effectiveness of the proposed algorithm.

\section{Acknowledgment}

The authors wish to express their gratitude to the anonymous reviewers and the associate editor for their rigorous comments during the review process. In addition, authors also would like to thank SUN Chengbin and TAN Lei in our laboratory for their great contributions to the data-collection work. This work was supported by the National Natural Science Foundation of China (Grant Nos. 61571014 and 61601006), Beijing Nature Science Foundation (Grant No. 4172017), and Beijing Municipal Science and Technology Project (Grant No. Z161100001016003).

Open Access This article is distributed under the terms of the Creative Commons Attribution 4.0 International License (http://creativecommons.org/licenses/by/4.0/), which permits unrestricted use, distribution, and reproduction in any medium, provided you give appropriate credit to the original author(s) and the source, provide a link to the Creative Commons license, and indicate if changes were made.

\section{References}

[1] W. Q. Lu, W. Liang, L. B. Zhang, and W. Liu, "A novel noise reduction method applied in negative pressure wave for pipeline leakage localization," Process Safety and Environmental Protection, 2016, 104(part A): 142-149.

[2] K. Jing and Z. H. Zou, "Time prediction model for pipeline leakage based on grey relational analysis,"
Physics Procedia, 2012, 25(2): 2019-2024.

[3] W. Liang, L. L. Lu, and L. B. Zhang, "Coupling relations and early-warning for 'equipment chain' in long-distance pipeline," Mechanical Systems and Signal Processing, 2013, 41(1-2): 335-347.

[4] X. Wang and M. S. Ghidaoui, "Identification of multiple leaks in pipeline: linearized model, maximum likelihood, and super-resolution localization," Mechanical Systems and Signal Processing, 2018, 107: 529-548.

[5] M. Zadkarami, M. Shahbazian, and K. Salahshoor, "Pipeline leakage detection and isolation: an integrated approach of statistical and wavelet feature extraction with multi-layer perceptron neural network (MLPNN)," Journal of Loss Prevention in the Process Industries, 2016, 43: 479-487.

[6] G. Allwood, G. Wild, and S. Hinckley, "Optical fiber sensors in physical intrusion detection systems: a review," IEEE Sensors Journal, 2016, 16(14): 5497-5509.

[7] Y. Zhan, Q. Yu, K. Wang, F. Yang, Y. Kong, and X. Zhao, "A high performance distributed sensor system with multi-intrusions simultaneous detection capability based on phase sensitive OTDR," Opto-Electronics Review, 2015, 23(3): 187-194.

[8] Y. Shi, H. Feng, Y. An, X. Geng, and Z. M. Zeng, "Research on wavelet analysis for pipeline pre-warning system based on phase-sensitive optical time domain reflectometry," in Proceeding of IEEE/ASME International Conference on Advanced Intelligent Mechatronics (AIM), Besancon, France, 2014, pp. 1177-1182.

[9] H. Q. Qu, T. Zheng, F. K. Bi, and L. P. Pang, "A vibration detection method for optical fiber pre-warning system," IET Signal Processing, 2016, 10(6): 692-698.

[10] Z. Z. Qiu, T. Zheng, H. Q. Qu, and L. P. Pang, "A new detection method based on CFAR and DE for OFPS," Photonic Sensors, 2016, 6(3): 261-267.

[11] N. Lu, B. W. An, and Y. L. Li, "Signal recognition algorithm of fiber-optic security system based on time-domain features," Transducer and Microsystem Technologies, 2017, 36(4): 150-152.

[12] L. Zhu, Z. M. Zeng, J. C. Zhang, and S. J. Jin, "Feature extraction of vibration signal detected by optical fiber along crude oil pipeline and forewarning system based on ICA," in Proceeding of International Workshop on Intelligent Systems and Applications, Wuhan, China, 2009, pp. 1-4.

[13] H. J. Wu, Z. N. Wang, F. Peng, Z. P. Peng, X. Y. Li, $\mathrm{Y}$. Wu, et al., "Field test of a fully distributed fiber optic intrusion detection system for long-distance 
security monitoring of national borderline," Society of Photo-Optical Instrumentation Engineers, 2014, 9157: 915790-915790-4.

[14] A. Klar and R. Linker, "Feasibility study of the automated detection and localization of underground tunnel excavation using Brillouin optical time domain reflectometer," SPIE, 2009, 7316(5): 433-443.
[15] Y. X. Lv, S. X Sun, and X. H. Gu, "Battlefield acoustic target classification and recognition based on EMD and power ratio," Journal of Vibration and Shock, 2008, 27(11): 51-55.

[16] Y. Lu, Z. K. Han, and Y. Chen, "FFT narrow band filtering method based on energy-ratio pretreatment," Journal of Southeast University (Natural Science Edition), 2010, 40(5): 948-951. 